\title{
CABRI test events monitoring through three measurement systems
}

\author{
Quentin Grando ${ }^{1}$, Léna Lebreton ${ }^{1}$, Vincent Chevalier ${ }^{1}$, Jacques Di Salvo ${ }^{1}$, Stéphane Eymery ${ }^{1}$, Claude \\ Gaillard $^{1}$, Nathalie Monchalin ${ }^{1}$, Jérôme Guillot ${ }^{1}$ \\ ${ }^{1}$ Nuclear Safety and Radiation Protection Institut - IRSN, France \\ quentin.grando@irsn.fr
}

\begin{abstract}
The CABRI experimental pulse reactor, located at the Cadarache nuclear research center, southern France, is devoted to the study of Reactivity Initiated Accidents (RIA). When the thermal-hydraulic conditions representative of a pressurized water nuclear reactor are reached (mostly temperature, pressure and flowrate conditions), a fuel test rod is submitted to a power excursion, triggered by the specific ${ }^{3} \mathrm{He}$ reactivity injection system, in order to simulate a control rod ejection accident. The experiment, managed by IRSN, aims at studying both the fuel and cladding behavior of the test rod placed into the center of the reactor during the power excursion.
\end{abstract}

Several test rods pre-irradiated in pressurized nuclear power plants and with different characteristics (burn up, cladding material, fuel type, Zirconia thickness) are considered for the programs performed in the CABRI reactor.

Physical phenomena occurring during the power transient are monitored by various measuring systems designed or operated by IRSN. Each system provides information linked with the different phases of the experiment. Three main measuring systems will be considered in this paper:

- The test devices, a sample holder that is also implemented with almost fifty sensors used to monitor the environmental parameters in the test channel such as temperature and pressure, and to control the rod behavior during the test sequence;

- The Hodoscope, an online fuel motion measurement system, which aims at analyzing the fuel motion deduced from the detection of fast neutrons emitted by the test rod, with a time step of $1 \mathrm{~ms}$ during the transient;

- The IRIS facility, conceived to perform X-ray radiography and tomography imaging before and after a power transient thanks to a linear electron accelerator, as well as quantitative gamma scanning analyses.

During the experimental sequence, different events are recorded. This paper focuses on a cladding failure that occurred during the transient and that can be revealed by the three systems mentioned above. The test device instrumentation allows to determine the timing of the failure and to analyze its consequences in the vicinity of the test rod from a thermal and hydraulic point of view, while the hodoscope measures fuel elongation and relocation during the power excursion.
The IRIS facility, then, helps to confirm the failure, its location and its extent. These three systems are complementary and they allow the analysis of the same event from different perspectives. Their combination will ease the interpretation of the events in the next steps for the test results analysis. The study case taken into account in this paper concerns nuclear fuel after three irradiation cycles in a commercial PWR.

Keywords - CABRI, Reactivity Initiated Accident, Test Device, Hodoscope, IRIS.

\section{INTRODUCTION}

$\mathrm{F}^{\mathrm{o}}$ OR enhancing safety of nuclear power plants (NPP), the Nuclear Safety and Radiation Protection Institute (IRSN) carries out some experimental programs in order to improve the understanding of the fuel behavior under severe accident conditions. Experiments are then driven into the CABRI experimental reactor, to test fuel sample under PWR conditions and submitted to a RIA accident.

The postulated design-basis reactivity accident for PWR is initiated by the ejection of a control rod bundle following a control rod housing rupture. For simulating the subsequent neutronic transient, the key feature of the CABRI reactor is its helium-3 reactivity injection system [1], which generates the power transients starting from a max $100 \mathrm{~kW}$ initial power (HZP - Hot Zero Power conditions). It is composed of 96 tubes located within the core, replacing the same number of fuel rods, grouped in 4 so-called "transient rods". These transient rods can be pressurized at up to 15 bar of helium-3, a strong neutronic absorber gas. These rods are all linked and gather in a collector. The depressurization towards a $1000 \mathrm{~L}$ discharge tank is made through two discharge lines (low and high flow rates), each one being equipped with a fast-opening valve followed by a controlled valve. A specific control command application is operated from the remote-control room. The helium-3 ejection leads to a reactivity injection (up to 3.9 \$) in a short time $(<80 \mathrm{~ms})$. The transient characteristics (Full Width at Half Maximum - FWHM, energy, power) depend on the sequence applied to the two fast-opening valves:

- Short FWHM $(<10 \mathrm{~ms}$ ) and high power (up to $30 \mathrm{GW}$ ) transients are obtained by opening a unique fast valve (the high flowrate channel one); 
- 20 to $80 \mathrm{~ms}$ FWHM high power transients (up to $10 \mathrm{GW}$ ) are obtained by successive and accurate opening of the two fastopening valves.

Results shown in this article will concern the d-day experiment. The reactor reach $100 \mathrm{~kW}$ in steady state condition with helium-3 into the transient rods, the experiment is triggered and the power excursion arises. The reactor is scrammed to end the chain reaction.

In order to analyse the test rod behaviour and collect data, the CABRI facility is equipped with various measurement systems. Among them, three systems are very specific and designed or operated by IRSN teams.

The first one is the CABRI test device. It holds the test rod and is also equipped with a variety of sensors that allow to record several thermal and hydraulic parameters of the system (such as temperature, pressure and flowrate) in the channel where the test rod is placed, as well as some other more exotic sensors used to measure more complex phenomena (like microphones, transient flowmeters, etc...).

The hodoscope, the second main measuring system coupled with the CABRI reactor, is a unique online fuel motion monitoring system, dedicated to the measurement of the fast neutrons emitted by the tested rod during the power pulse. This system is used to observe the degradation of the fissile column, and is able to measure fuel motion, potentially linked to clad failure, if it arises during the power excursion.

The third equipment is the IRIS (Installation for radiography, imaging and spectrometry) facility dedicated to pre and post non-destructive examinations of the rodlet. This measuring system is equipped by two main parts:

- A gamma spectrometry system which is used to measure gamma rays emitted by the fission products in the test rod.

- An X-ray facility which aims at performing X-ray radiography and tomography images.

The next section of this article gives a brief description of the three systems. Section four provides simplified test data showing how the three systems are complementary to describe the events that occur during the experimental phase.

\section{MEASUREMENT SYSTEMS OVERVIEW}

\section{A. Test device description}

\section{1) General description of the system}

To achieve the test objectives, a specific equipment was designed by IRSN. The test device acts as test rod holder. It allows to maintain it in place, at the right elevation at the center of the reactor core. It also allows water from the water loop at PWR conditions to run through the test channel where the test rod is placed. The primary water also runs around the test device in order to cool the outside walls of the device as well as the inner walls of the loop. Instrumentation is embedded in it. Mainly dedicated to measure thermal and hydraulical parameters such as flow rate, pressure and temperature into the test channel, it also includes various specific sensors much more dedicated to investigate the phenomenology of the CABRI tests.
In this paper, a focus will be made on the measurements of the temperature in the vicinity of the rod and the flowrate at the moment of the interaction between hot fuel and water. The test device is made of three specific parts, detailed from the bottom to the top in the following paragraphs.

The first part is the lower part. It is placed below the flange that allows to insert the test rod. This part especially holds the upstream transient flowmeter.

The next one is the core zone where the test rod is held. It is made of Zircaloy for neutrons transparency purpose. Only thermocouples are implemented in this area. The most important of them are the twelve sensors that are placed in the vicinity of the test rod into the channel, and held by a spacer system.

The last part is the upper part. At its upper end stands the water outlet. The downstream transient flowmeter and a rod elongation sensor are implemented there. Some more specific sensors are also implemented but not detailed in this article. Above the water outlet is the head of the test device. It allows to close tightly the pressurized water cell and also holds the connectors that route the signals to the conditioners and the acquisition systems.

The following figure, extracted from [1], shows an overview of the test device with a focus on the implemented sensors.

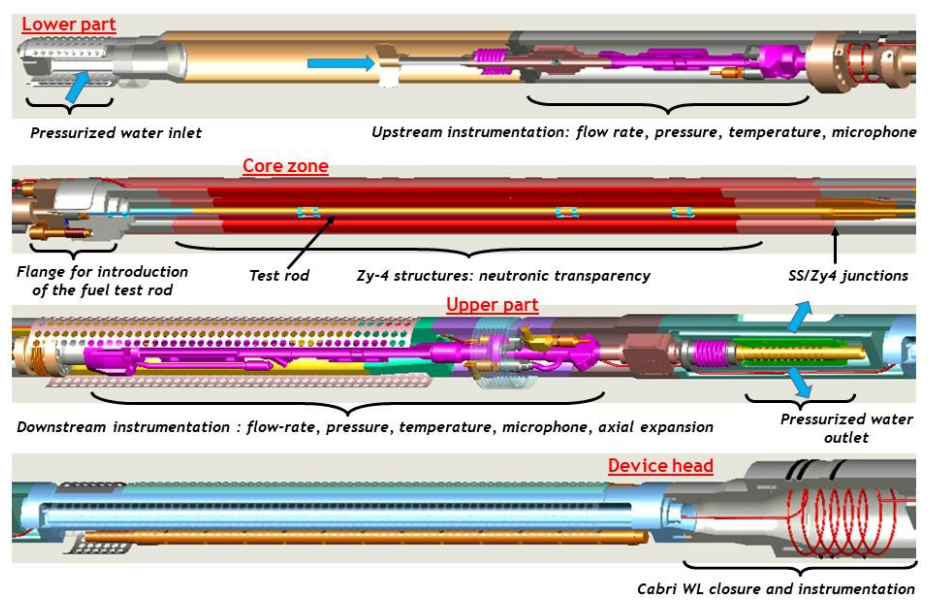

Fig. 1. Test device simplified description.

\section{2) Instrumentation implemented in the device}

The key sensors are located upstream and downstream the test rod to avoid being in the core zone, except for twelve Ktype thermocouples that are placed in its vicinity. The rod is centered on the core axis and has a total length of around $1.1 \mathrm{~m}$ with a $\sim 56 \mathrm{~cm}$ long fissile column.

The thermocouples are implemented on a structure called spacer system that allows the rod being centered and the thermocouples being placed at the right axial location along the rod length. They are distributed along the fissile column to measure the fluid temperature in the vicinity of the rod.

The flowmeters of interest in this paper are the two fast response flowmeters, based on the LVDT technology. They are located upward and downward the rod. 


\section{B. Hodoscope description}

The CABRI reactor has been equipped with the hodoscope since 1977. This system measures in real time the behaviour of the test rod in the center of the CABRI core during the transient. The hodoscope is able to quantify the amount of fuel ejected in the milliseconds following failure, time-dependent axial fuel mass distributions and follows the fuel clusters after failure. In addition, the initial state of the test rod is precisely known thanks to the hodoscope measurement of the fissile length and the axial power profile.

The hodoscope centerpiece is a $3 \mathrm{~m}$ steel collimator (in green in Fig. 2) of 3 columns and 51 rows, making 153 channels. Behind each one of these line-of-sights, $\mathrm{a}^{237} \mathrm{~Np}$ fission chamber detector (FC) and a proton recoil proportional detector (PR) measure the fast neutrons coming from the core and test rod. Two different technologies of detectors are used in CABRI in order to follow all the experiments from low core power $(\sim 50 \mathrm{~kW})$ up to $20 \mathrm{GW}$. Proton recoil counters are better suited for low power (up to $\sim \mathrm{GW}$ which means 2.5 million impulses per second), thanks to a higher efficiency and no discrimination of the $\mathrm{y}$ noise, whereas ${ }^{237} \mathrm{~Np}$ fission chambers are used for higher power. The signal acquisition rate can be adjusted between $1 \mathrm{~ms}$ and $10 \mathrm{~s}$ according to the count rate. The associated electronics have been renewed taking benefit from the renovation period [2].

The collimator was built in order to pixelate the test rod placed in the center of the reactor. Each detector looks at a slice of the test rod. In steady state conditions, the detectors measure the axial power profile of the test rod, whereas in transient conditions (i.e. a RIA pulse) the signals vary according to a fuel displacement, a fuel densification (yielding to an increasing signal), or a fuel dilution or ejection (yielding to a decreasing signal). It should be noticed that two lines of sights, row 17 and 35 , are filled with stainless steel in order to mechanically strengthen the collimator.

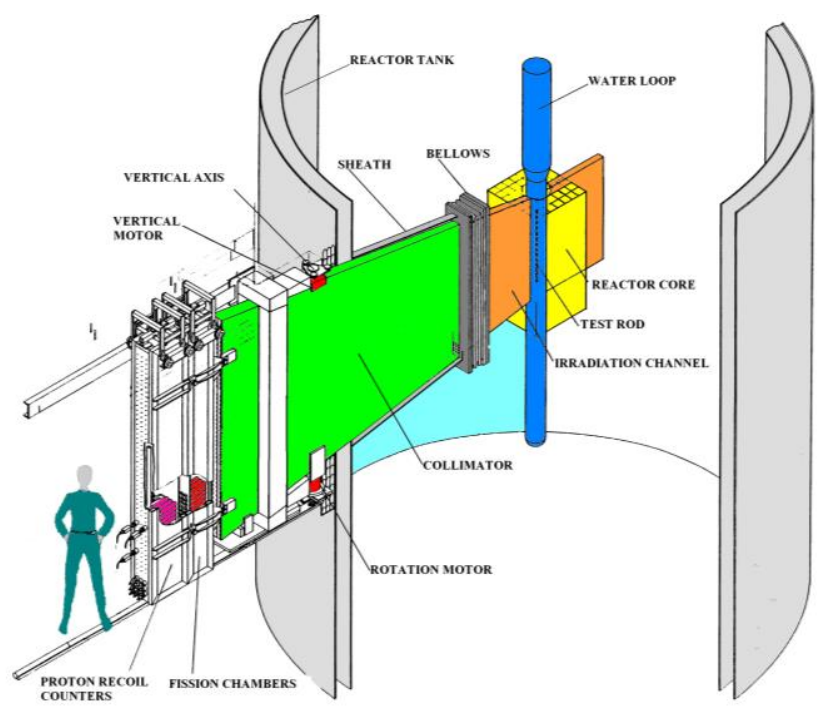

Fig. 2. Hodoscope detector system.

\section{IRIS facility}

The IRIS facility (Installation for Radiography, Imaging and Spectrometry, Fig. 3) is a non-destructive examination bench. IRIS can perform three types of examination:

- Quantitative gamma-scanning.

- X-ray transmission radiography,

- X-ray transmission tomography.

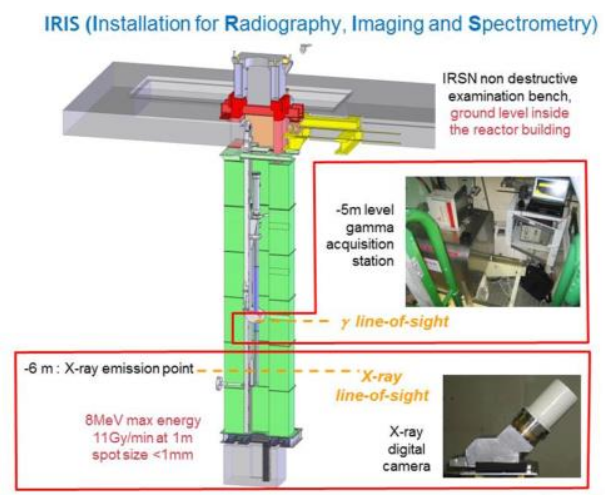

Fig. 3. IRIS facility.

Located within the CABRI reactor building, the mechanical bench is installed in vertical position, for avoiding modifying the post-test final state of the rod, inside a concrete and iron radiological shielding. The test device is placed within a tightness sheath that is motorized in vertical translation and in rotation in front of two examination line-of-sights.

Quantitative gamma-scanning is performed with vertical translation in front of a $1 \mathrm{~mm}$ height collimation. The detector is a high purity Germanium detector, coupled to a remotecontrolled acquisition system. Before the test, the distribution of these radionuclides allows to verify the integrity of the fissile column. The results of gamma spectrometry will not be developed here.

$\mathrm{X}$-ray radiographies and tomographies are carried out by means of a NMOS camera. The X-ray beam is generated by a high energy electrons linear accelerator (the maximum energy reaches $8 \mathrm{MeV}$, mean energy is around $2.5 \mathrm{MeV}$ ), producing a dose rate of more than $10 \mathrm{~Gy} / \mathrm{min}$ at $1 \mathrm{~m}$. Radiographies are acquired by vertical translation of the device in front of the camera, equipped with a $\sim 0.5 \mathrm{~mm}$ entry slot collimator, while tomographies combine step-by-step rotation $\left(0.4^{\circ}\right.$ azimuthal step) at a given elevation. The spatial resolution of the reconstructed images is close to $200 \mu \mathrm{m}$.

Before the test, from the radiography acquired at $0.5 \mathrm{~mm}$ step, the bottom and the top of the fissile column (BFC and TFC) are determined and compared to the ones measured by gamma spectrometry. The fissile column length is then confirmed. The location and integrity of the device sensors are verified also from radiographic examination.

The tomographies at positions chosen in relation to the zones of interest determined by radiography, allow to carry out a sectional view of the rod. When the density of the material crossed by the rays becomes greater, the image appears blacker. Before the test, these images make it possible to check the centering of the rod in the channel tube, the integrity of the spacer rings and the position of the sensors. 
The tomographies also make it possible to measure the diameter of the rod before and after the test and thus to evaluate a possible rod swelling due to the transient. The dimensional measurements are made relative to the known values of the channel tube and the pressure tube diameters.

\section{RESULTS}

Several noticeable events were observed during the transient. For this article, focus will be made on the event arising at the time TOP ONSET $+379 \mathrm{~ms}$ (Transient Over Power ONSET, which will be noted t0, corresponds to the beginning of the neutron population rise), leading to a clad failure on the top part of the test rod.

Fig. 4 summarizes the main characteristics of the pulse and illustrates the time interval of interest in the test sequence: the three measurement systems will be further analyzed within this interval. It runs from $\mathrm{t} 0+375 \mathrm{~ms}$ to $\mathrm{t} 0+392 \mathrm{~ms}$.

They all will be taken from the point of view of their own specific skills allowing them to monitor specific parameters evolution, but also from their ability to complement each other and widen the view of the monitored events.

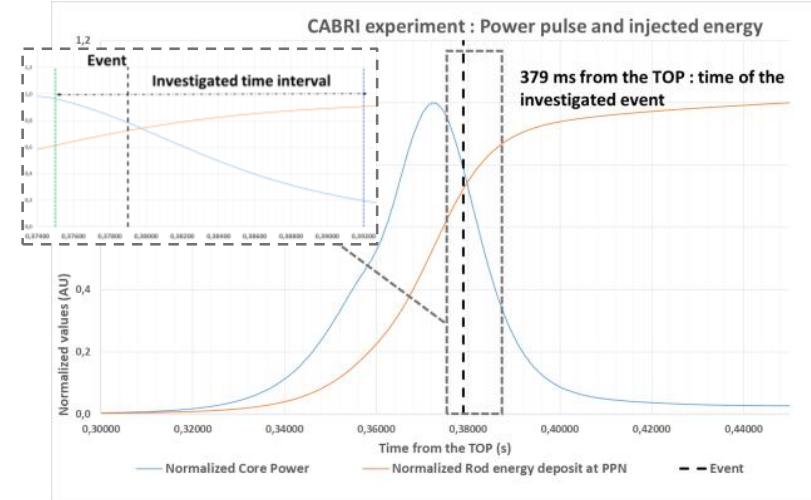

Fig. 4. Core power and rod energy deposit during the pulse.

\section{A. Measurements from the test device instrumentation point of view}

As already mentioned, a clad failure event occurs around $379 \mathrm{~ms}$ after TOPONSET.

The first sensor that detects the event is the DA, with a fast, but limited, decrease of the normalized elongation followed by a return to a different baseline (Fig. 5).

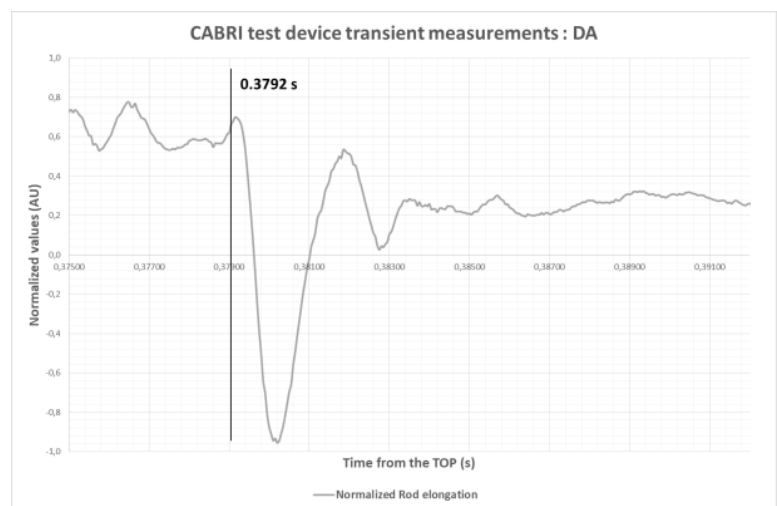

Fig. 5. Test device measurements of interest: elongation (DA).

The event can then be detected with the transient flow rates sensors QT1 \& 2 (379.5 ms on Fig. 6). They show opposite variations that are a sign of water ejection in the two available directions. QT2 catches the event $0.7 \mathrm{~ms}$ before QT1 (this timing is obtained by the use of the moving variance technique, but it's difficult to associate a uncertainty to this value), indicating that this event occurs closer to the top of the rod.

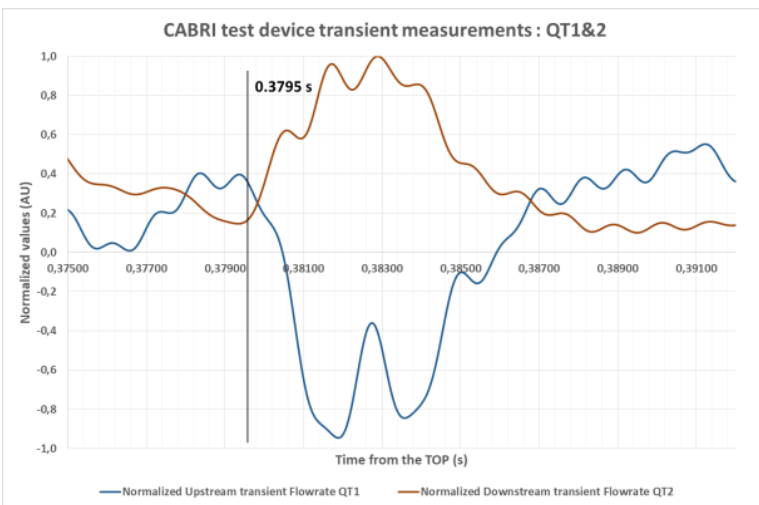

Fig. 6. Test device measurements of interest: transient flowrate (QT1\&2).

The behavior of the sensors shown in this paragraph tends to confirm that a fuel-coolant interaction occurred. The time difference between downstream and upstream sensors tends to indicate that the event occurred in the upper part of the rod. A two phase mixture can also be guessed with one of the TCs placed on the rod cladding (not shown in this paper), that expresses a very high increase of the clad temperature from $372.5 \mathrm{~ms}$ after the top, marking a possible boiling. This information and the fact that only one TC reached a so high temperature tends also to indicate that both the water state and the temperature distribution are not homogeneous in the channel along the rod, which increases the difficulties to give a precise date and location of the event.

\section{B. Hodoscope and test device data collected during the transient}

1) Accumulation of fuel detected by the hodoscope equipment

One of the most important measurement performed by the hodoscope is the evolution of the axial fuel distribution during the power transient for each hodoscope row. The measurements are based on the count rates of the ${ }^{237} \mathrm{~Np}$ fission chambers (fission threshold at $600 \mathrm{keV}$ ), better suited for the high level power reached during the transient. As already mentioned, for each row of detectors, only fast neutrons are detected by the hodoscope sensors. Thanks to the acquisition rate with a time step of $1 \mathrm{~ms}$, it is possible to determine the moment and the characteristics of the degradation.

We define [4]:

- $\mathrm{S}_{\mathrm{i}}(\mathrm{t})$ : signal created on row $\mathrm{i}$ by fast neutrons coming directly from the test rod at time $t$.

- $\quad \mathrm{B}_{\mathrm{i}}(\mathrm{t})$ : the so-called « noise background » created by fast neutrons coming from the core and fast neutrons scattered by the test loop and detected on row $\mathrm{i}$ at time t.

- $\mathrm{SNR}_{\mathrm{i}}(\mathrm{t})$ : the Signal over Noise Ratio $\mathrm{SNR}_{\mathrm{i}}(\mathrm{t})=$ $\mathrm{S}_{\mathrm{i}}(\mathrm{t}) / \mathrm{B}_{\mathrm{i}}(\mathrm{t})$. 
- $\quad \mathrm{SNR}_{\mathrm{i}}(\mathrm{BT})$ : the average $\mathrm{SNR}$, recorded during the power plateau at $100 \mathrm{~kW}$ just before the transient (Before TOP onset) .

As the variations of $\mathrm{SNR}_{\mathrm{i}}(\mathrm{t})$ are very small, the analysis system computes a normalized Signal over Noise Ratio, $\mathrm{SNR}_{\mathrm{i}}(\mathrm{t}) / \mathrm{SNR}_{\mathrm{i}}(\mathrm{BT})$. This is the quantity to which the signal to fuel mass conversion abacuses can be applied [5].

The hodoscope system plots the evolution of $\mathrm{SNR}_{\mathrm{i}}(\mathrm{t}) / \mathrm{SNR}_{\mathrm{BT}}$ during the power plateau and the power transient.

$\mathrm{SNR}_{i}(\mathrm{t})>\mathrm{SNR}_{i}(\mathrm{BT})$ or $\mathrm{SNR}_{i}(\mathrm{t})<\mathrm{SNR}_{i}(\mathrm{BT})$ means a fuel displacement during the power transient in the $i^{\text {th }}$ row.

In this article, the rows 14 to 30 corresponding to the top part of the fuel rod are particularly considered. To understand the Fig. 7, one have to know: a ratio $\mathrm{SNR}_{\mathrm{i}}(\mathrm{t}) / \mathrm{SNR}_{\mathrm{i}}(\mathrm{BT})>1$ will appear in green, corresponding to a fuel accumulation; on the contrary, a ratio $\mathrm{SNR}_{\mathrm{i}}(\mathrm{t}) / \mathrm{SNR}_{\mathrm{i}}(\mathrm{BT})<1$ will be represented in red, meaning a lack of fuel. The blue curve overriding the different channels corresponds to the power level measured by the experimental chambers of the CABRI reactor. The grey dashed rectangle delimits the time interval [t0+375 ms; t0+392 ms] during which the failure occurred in the upper part of the fuel rod.

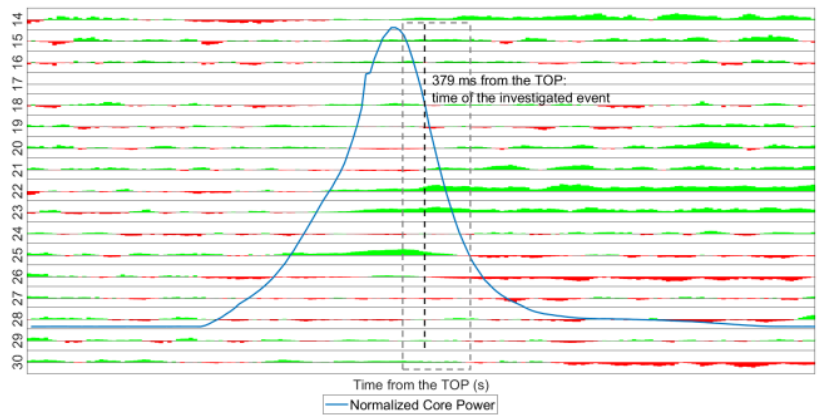

Fig. 7. Variation of the S/B before and during power excursion measured by the hodoscope's detectors for the upper part of the fuel rod (rows 14 to 30).

During and at the end of the transient, one can note some rows for which accumulation of fuel material is observed (row 14 linked with the $\sim$ top of the fuel rod, rows below 15, 20, 21, 22, 23 ) and others for which lack of fuel can be noticed (rows 25 , 26,28 corresponding to the $\sim$ middle of the fuel rod, and row 30 ). Accumulation of fuel for the rows 20 to 23 corresponds to an axial zone located between -120 to $-200 \mathrm{~mm}$ below the TFC.

\section{2) Clad failure detected by the test device instrumentation}

Focusing on the time interval between [t0+375 ms; t0+392 ms] on Fig. 8, in the test channel, the large flowrate divergence is observed (transient flowmeters QT1 and QT2) and superposed to hodoscope results.

After the event, the hodoscope recorded a continuous fuel relocation in the upper part of the test rod.

Therefore, transient flowmeters signals combined with hodoscope measurements may reveal clad failure.

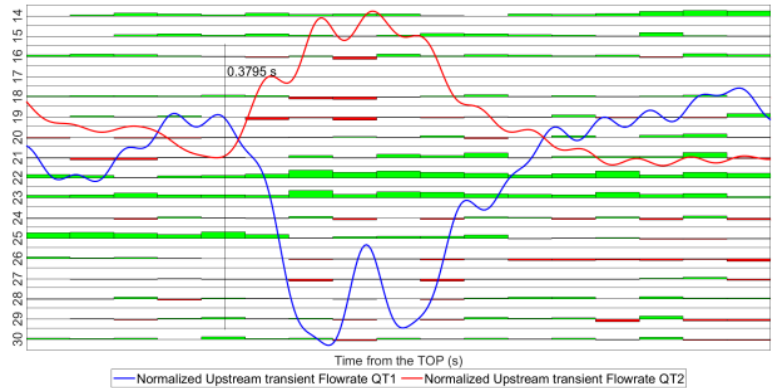

Fig. 8. Variation of signals measured by the device instrumentation (flowmeters) and S/B ratio evolution obtained with the hodoscope detectors for the upper part of the fuel rod, for a time range [t0+375ms; $0+392 \mathrm{~ms}]$.

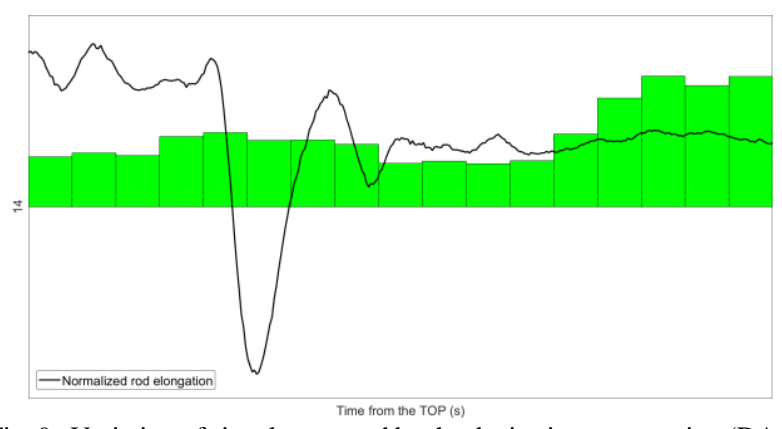

Fig. 9. Variation of signal measured by the device instrumentation (DA sensor) and S/B ratio evolution deduced from the hodoscope detectors for the row 14, for a time range $[\mathrm{t} 0+375 \mathrm{~ms} ; \mathrm{t} 0+392 \mathrm{~ms}$.

Consistent with the event detected by the axial expansion sensor DA, the hodoscope row 14 reveals the elongation of the fissile column (Fig. 9).

\section{Hodoscope and IRIS data collected after the transient \\ 1) Final state of the fuel rod detected by the hodoscope equipment}

The final state of the rod after the transient is measured during a power plateau at $100 \mathrm{~kW}$ for 1 hour (in order to get enough statistics), starting $\sim 75$ minutes after the power transient. The fuel distribution is estimated as the ratio of the post-test signal to the pre-test signal.

The results obtained are presented for both PR and FC sensors. Fuel accumulation appears in green, while fuel loss is traced in red. The accumulation zone is evidenced in the upper part, and the lack of fuel is observed in the center part.
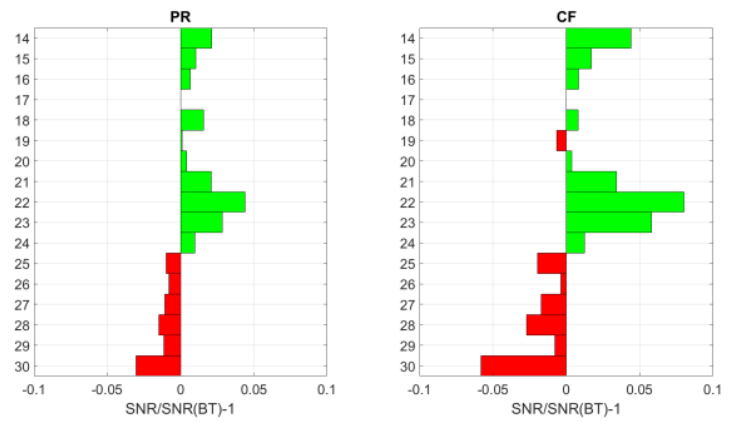

Fig. 10. Final state of the upper part of the fuel rod deduced from the hodoscope's detectors (rows 14 to 30 ). 


\section{2) Final state of the fuel rod obtained with IRIS X-ray measurements}

The X-ray results are presented. Fig. 11 compares the radiography of the test rod performed before the transient (on the left, with only one angular position shown because the geometry was axisymmetric), to the three other ones performed after the test at three different angles: $\Theta^{\circ}, \Theta+60^{\circ}$ and $\Theta+120^{\circ}$ (on the right). The density and thickness of the materials crossed by the X-rays lead to blurring of the image (scattering of the rays). Concentric circles appear and define, from the center outwards, the pellet delimited by the clad, the space between the rod and the channel tube, the channel tube, the space between the channel tube and the pressure tube and finally the pressure tube. Small dots indicate the presence of sensors in the space between the rod and the channel tube and in the space between the channel tube and the pressure tube.

Ejection of fuel led to lack of fuel at some places and accumulation at other places. A zone of fuel accumulation is roughly located between $-110 \mathrm{~mm}$ and $-180 \mathrm{~mm}$ below the TFC, in consistency with hodoscope results.

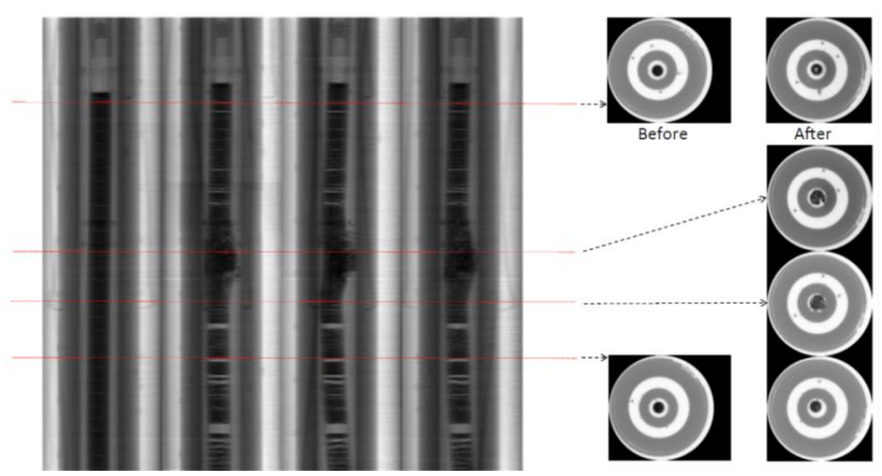

Fig. 11. Final state of the upper part of the fuel rod obtained with the X-ray measurements of IRIS equipment.

The rod is clearly not centered towards the vertical axis on the tomographies: a S-shaped deformation of the rod is observed. After the test, a residual elongation of the fissile column of at least $8 \mathrm{~mm}$ is observed. Radiographies show a clear crushing of the spring, which length is reduced of $\sim 8 \mathrm{~mm}$ after the test. The top end of the upper plug did not move axially. The spacer rings are identified at the same location before and after the test.

On the tomographies after the test, the clad is clearly opened on the upper part of the rod. Fuel has been ejected, fuel fragments are located between the clad and the channel tube. The fuel remaining in the clad shows a fragmented structure.

The tomographies make it possible to locate the angular position of the clad opening. The combination of radiography and tomography allows to define the length of the opening.

\section{3) Combination of the results}

One has to be very careful when comparing the results of the different equipment in terms of axial localization, because the conditions of the measurements are quite different: hot (PWR) conditions for hodoscope measurements and cold (room temperature) for IRIS measurements.

However, we can observe on Fig. 12 the very good consistency obtained for qualitative results coming from these two equipments: the hodoscope PR measurements (on the left) and the X-radiography (on the right). At the location of the clad failure, the accumulation of fuel is clearly visible at the same level. One must keep in mind that for the hodoscope sensors, the results are expressed in relative terms, i.e. positive values means that, at the final state, more fuel is detected at the sight of view of the row than before the pulse. The results can be obtained very quickly after the transient and helps to the first interpretation of the results in combination with the other sensors.

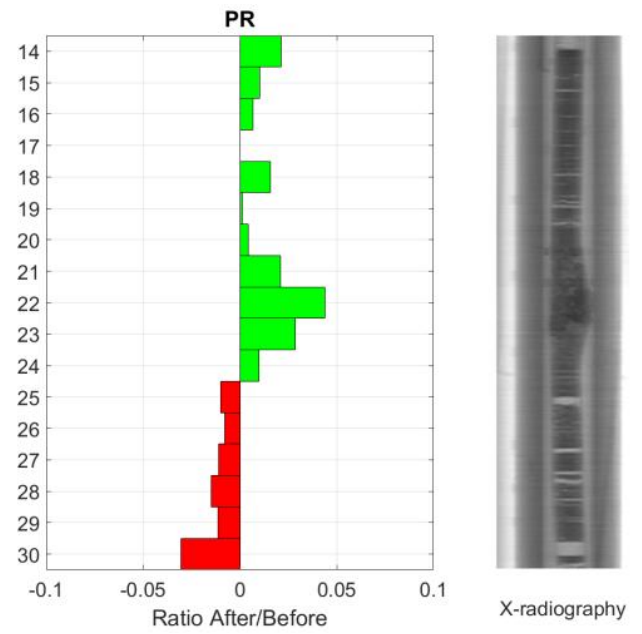

Fig. 12. Comparison of the final state of the upper part of the fuel rod obtained with the different devices.

\section{CONCLUSIONS}

In this paper, we highlighted that a sequence of events can be very precisely monitored from various and complementary points of view thanks to the different devices implemented in CABRI.

Each one has its own skills and monitors specific quantities. First, the test device sensors give information from a thermohydraulic point of view, allowing a timing of the events and raw location. The hodoscope then monitors it from the fuel point of view and, when combined with the test device sensors, sharpen timing and location estimation. They both give a view of the events occurred during the test sequence and allow also deeper analysis later after the test sequence once the raw data are processed. Finally, the IRIS equipment provides the final state of the fuel rod, confirming and localizing more precisely the event. The possible damages to the cladding or fuel stack can be further analyzed. The precious information produced is very helpful for the definition of Post Irradiation Examinations of the tested fuel rod in a hot laboratory.

\section{ACKNOWLEDGMENT}

The author express their acknowledgments to all the contributors of this project, and especially the experimental physicist team, the engineering team and the operators of the CABRI reactor working at the CEA.

\section{REFERENCES}

[1] B. Biard et al., "Reactivity Initiated Accident Transient on irradiated fuel rods in PWR conditions: the CABRI International Program CIP" Annals 
of Nuclear. Energy , vol. 141, 2020,

https://doi.org/10.1016/j.anucene.2019.107253.

[2] S. Mirotta et al., "Qualification and characterization of electronics of the fast neutrons Hodoscope detectors using neutrons from the CABRI core'" presented at the fifth ANIMMA conference, Liege, Belgium, June. 1923, 2017.

[3] V. Chevalier, S. Mirotta, J. Guillot: The CABRI fast neutron Hodoscope: renovation, qualification program and first results following the experimental reactor restart, "In ANIMMA2017 Conference Proceedings, Liege, June 2017.

[4] J. Di Salvo, S. Mirotta, V. Chevalier, "Analysis of the Signal over Noise Ratio of the hodoscope determined by Monte Carlo calculation", presented at the $7^{\text {th }}$ International Conference ANIMMA, June 21-25, 2021

[5] Baumung et Augier. Quantitative fuel motion determination with the CABRI fast neutron Hodoscope: Evaluation Methods and Results, november 1990. 